\title{
Disease Severity and Genotype Affect Physical Growth in Children With Familial Mediterranean Fever
}

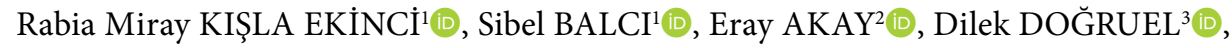 \\ Derya Ufuk ALTINTAŞ ${ }^{3}$ (D), Mustafa YILMAZ ${ }^{1}$ (D) \\ ${ }^{1}$ Department of Pediatric Rheumatology, Çukurova University Faculty of Medicine, Adana, Turkey \\ ${ }^{2}$ Department of Pediatrics, Çukurova University Faculty of Medicine, Adana, Turkey \\ ${ }^{3}$ Department of Pediatric Allergy and Immunology, Çukurova University Faculty of Medicine, Adana, Turkey
}

\begin{abstract}
Objectives: This study aims to analyze the growth parameters in children with familial Mediterranean fever (FMF) according to disease characteristics including genotype and disease severity by a recently validated tool in relatively more patients.

Patients and methods: This retrospective study included 126 patients with FMF (70 males, 56 females; mean age $7.3 \pm 3.6$ years; range, 4.1 to 18 years). MEditerranean FeVer (MEFV) gene analysis was performed with a molecular diagnostics tool by using a next-generation sequencing platform. Disease severity was determined for the first visit by the validated tool in children, international severity scoring system for FMF. Growth parameters including weight and height were investigated after standard deviation (SD) scores were calculated by anthropometric references in Turkish children. Results: Median follow-up duration was 74.7 months (range, 7.5 to 169 months). Ninety-three patients (73.8\%) had at least one M694V mutation in MEFV gene. Six patients (4.8\%) had severe disease, 58 (46\%) had intermediate severity, and 62 (49.2\%) had mild disease. Mean height SD score was significantly lower at last visit than before colchicine treatment. Initial and last height and weight SD scores were lower in patients with at least one M694V mutation than those without. However, the difference was statistically significant for only initial height SD score. We also found statistically significant lower initial height, final height, and weight SD scores in patients with intermediate severity-severe disease activity than mild disease.

Conclusion: We advise physicians to score disease severity prospectively and pay attention to patients with intermediate severity-severe disease to avoid growth disturbances.
\end{abstract}

Keywords: Disease severity; familial Mediterranean fever; growth; international severity scoring system for familial Mediterranean fever; M694V.

Familial Mediterranean fever (FMF) is the most common hereditary autoinflammatory disease characterized by recurrent attacks of fever, serositis, arthralgia or arthritis. ${ }^{1}$ More than 300 mutations were described in the causative MEditerranean FeVer (MEFV) gene so far. $^{2}$ Mutations in MEFV gene results in pyrin inflammasome and caspase-1 activation, thus increased interleukin 1 beta (IL-1ß) maturation and inflammation. FMF usually presents with a selflimited course and childhood-onset symptoms. ${ }^{3}$
Disease flares generally occur within variable intervals from once weekly to once a year and last for one to three days. ${ }^{1}$ The most terrifying complication of FMF is renal amyloidosis due to ongoing inflammation; fortunately, it was suggested that colchicine treatment decreased amyloidosis rate to $8.6 \%$ in a large FMF cohort from Turkey. ${ }^{4}$ Growth is an important indicator of well being in children and chronic inflammatory diseases are frequently known to lead to growth impairment. Moreover, several studies including

Received: September 25, 2018 Accepted: November 20, 2018 Published online: January 28, 2019

Correspondence: Rabia Miray Kışla Ekinci, MD. Çukurova Üniversitesi Tıp Fakültesi Pediatrik Romatoloji Anabilim Dalı, 01790 Sarıçam, Adana, Turkey. Tel: +90507 - 2171190 e-mail: mir_kisla@hotmail.com 
limited numbers of patients addressed the question whether physical growth is affected by genotype and disease severity or not. ${ }^{5-10}$ However, all these studies utilized an ancient Tel Hashomer severity scoring system, which was not validated in the pediatric population. ${ }^{11}$ Thus, in this study, we aimed to analyze the growth parameters in children with FMF according to disease characteristics including genotype and disease severity by a recently validated tool in relatively more patients.

\section{PATIENTS AND METHODS}

This study included 126 patients with FMF (70 males, 56 females; mean age $7.3 \pm 3.6$ years; range, 4.1 to 18 years) who were diagnosed according to Tel Hashomer criteria between August 2004 and January 2018 and followed-up for at least six months in Pediatric Rheumatology Department of Çukurova University Faculty of Medicine. Medical files were retrospectively reviewed for data including sex, age at onset, age at diagnosis, MEFV mutations, attack frequency, symptoms, acute phase reactants (erythrocyte sedimentation rate [ESR], C-reactive protein, and serum amyloid-A) in attack-free period, duration of colchicine treatment, colchicine response, disease severity scores, and growth parameters at diagnosis and at the end of follow-up. Disease severity was retrospectively assessed for the first visit, by the validated tool in children, international severity scoring system for FMF (ISSF). Patients were excluded if ISSF assessment or growth indices could not been utilized due to lack of eligible information. Disease severity was grouped as severe ( $\geq 6$ points), intermediate (3-5 points), or mild ( $\leq 2$ points)..$^{12}$ Growth parameters including weight and height were investigated; thereafter, standard deviation (SD) scores were calculated by anthropometric references in Turkish children. ${ }^{13}$ The study protocol was approved by the Çukurova University Faculty of Medicine Ethics Committee (number: 78/68; date: 01 June 2018). A written informed consent was obtained from parents of each patient. The study was conducted in accordance with the principles of the Declaration of Helsinki.

We utilized the MEFV gene analysis as a molecular diagnostics tool by using a next-generation sequencing platform (MiSeq System, Illumina, San Diego, CA, USA). Sequencing was performed in leukocyte deoxyribonucleic acid from all cases. In silico analysis was performed with Sorting Intolerant from Tolerant, PolyPhen-2, and Mutation Taster for novel mutations and also confirmed by paternal testing to identify the carrier status.

\section{Statistical analysis}

We used the IBM SPSS 20.0 software (IBM Corp., Armonk, NY, USA). Categorical variables were expressed as percentages, while continuous variables were summarized as mean and SD and as median and minimum-maximum where appropriate. The normality of distribution for growth indices was confirmed with the Kolmogorov-Smirnov test, stem-and-leaf diagram and the histogram. For comparison of continuous variables between two groups, the Student' t-test was used except for weight SD score before colchicine treatment. Due to the moderate skewness and kurtosis of the data, we compared weight SD scores with Mann-Whitney $U$ test between two groups. Additionally, paired-sample t-test was utilized for comparing two dependent variables in the same study group. The statistical level of significance for all tests was determined to be 0.05 .

Table 1. Clinical findings of 126 children with familial Mediterranean fever

\begin{tabular}{lcc}
\hline Manifestations & $\mathrm{n}$ & $\%$ \\
\hline Fever & 115 & 91.3 \\
Abdominal pain & 119 & 94.4 \\
Arthralgia & 89 & 70.6 \\
Arthritis & 15 & 11.9 \\
Chest pain & 8 & 6.3 \\
Nausea & 18 & 14.3 \\
Diarrhea & 6 & 4.8 \\
Erysipelas-like erythema & 11 & 8.7 \\
Prolonged febrile myalgia & 1 & 0.8 \\
Splenomegaly & 3 & 2.4 \\
Colchicine dosage & & \\
$\quad 0.5$ mg/day & 17 & 13.5 \\
$\quad 0.75$ mg/day & 2 & 1.6 \\
1 mg/day & 86 & 68.3 \\
1.5 mg/day & 21 & 16.7 \\
Colchicine resistance & 5 & 3.9 \\
\hline
\end{tabular}


Table 2. Growth parameters of 126 patients with familial Mediterranean fever before colchicine treatment and at last visit

\begin{tabular}{|c|c|c|c|}
\hline & Before colchicine treatment & At last visit-under colchicine treatment & \\
\hline & Mean \pm SD & Mean \pm SD & $p$ \\
\hline Weight SD score & $-0.24 \pm 1.31$ & $-0.45 \pm 1.22$ & $0.087^{*}$ \\
\hline Height SD score & $0.13 \pm 1.45$ & $-0.32 \pm 1.32$ & 0.006* \\
\hline
\end{tabular}

\section{RESULTS}

Median follow-up duration was 74.7 months (range, 7.5-169 months). Clinical manifestations of the patients were summarized in Table 1. Ninety-three patients (73.8\%) had at least one M694V mutation in MEFV gene. Six patients (4.8\%) had severe, 58 (46\%) had intermediate, and $62(49.2 \%)$ had mild disease according to initial ISSF scoring. We concomitantly treated five patients with canakinumab, who were determined to be colchicine resistant. They were attack-free and had normal acute phase reactants at the time of study.

Weight and height SD scores were compared before colchicine initiation and at last visit. Mean height SD score was significantly lower at last visit than prior to colchicine treatment (Table 2). Furthermore, patients were grouped according to their initial ISSF scores as following: mild disease

Table 3. Comparison of growth parameters regarding presence of mild disease or intermediate severity-severe disease according to ISSF scoring

$\begin{array}{lcccc} & \text { Mild disease }(\mathrm{n}=64) & & \text { Intermediate severity-severe disease }(\mathrm{n}=62) \\ \text { Growth parameter } & \text { Mean } \pm \text { SD } & & \text { Mean } \pm \text { SD } & p \\ \begin{array}{l}\text { Before colchicine treatment } \\ \text { Weight SD score }\end{array} & -0.17 \pm 1.15 & & -0.31 \pm 1.46 & 0.217^{*} \\ \quad \text { Height SD score } & 0.40 \pm 1.29 & & -0.13 \pm 1.55 & \mathbf{0 . 0 3 8} \# \\ \text { At last visit-under colchicine treatment } & & & -0.75 \pm 1.14 & \mathbf{0 . 0 0 5} \# \\ \quad \text { Weight SD score } & -0.15 \pm 1.23 & & -0.58 \pm 1.31 & \mathbf{0 . 0 2 4} \# \\ \text { Height SD score } & -0.05 \pm 1.29 & & \end{array}$

ISSF: International severity scoring system for familial Mediterranean fever; SD: Standard deviation; * Mann-Whitney $U$ test was used for data comparison; \# Student's t-test was used for data comparison; Significant $p$ values $(<0.05)$ are in bold.

Table 4. Comparison of growth parameters between patients with or without at least one M694V mutation on MEFV gene

\begin{tabular}{|c|c|c|c|}
\hline & $\begin{array}{l}\text { Patients with at least one M694V } \\
\text { mutation }(n=93)\end{array}$ & $\begin{array}{l}\text { Patients with mutations other than } \\
\text { M694V (n=33) }\end{array}$ & \\
\hline Growth parameter & $\begin{array}{l}\text { Mean } \pm \text { SD } \\
\end{array}$ & Mean \pm SD & $p$ \\
\hline \multicolumn{4}{|c|}{ Before colchicine treatment } \\
\hline Weight SD score & $0.05 \pm 1.19$ & $-0.35 \pm 1.34$ & $0.044^{*}$ \\
\hline Height SD score & $0.59 \pm 1.29$ & $-0.03 \pm 1.47$ & $\mathbf{0 . 0 2 3} \#$ \\
\hline \multicolumn{4}{|c|}{ At last visit-under colchicine treatment } \\
\hline Weight SD score & $-0.27 \pm 1.07$ & $-0.51 \pm 1.26$ & $0.299 \#$ \\
\hline Height SD score & $-0.07 \pm 1.04$ & $-0.41 \pm 1.41$ & $0.160 \#$ \\
\hline
\end{tabular}


(ISSF score $\leq 2)$ and intermediate severity-severe disease (ISSF score $\geq 3$ ). The growth parameters were compared between these two groups in Table 3.

Additionally, we compared the growth parameters between patients with at least one M694V mutation on MEFV gene and those without. Initial and last height and weight SD scores were lower in patients with at least one M694V mutation than those without. However, the difference was statistically significant for only initial height SD score (Table 4).

\section{DISCUSSION}

Diagnosis of FMF is still based on clinical parameters; however, genetic studies are used for confirming, particularly in case of any doubt in the clinical diagnosis. Homozygosity of M694V mutation in MEFV gene was related to a severe phenotype in several studies. ${ }^{14-16}$ The major goals of FMF management are complete control of attacks and preventing subclinical inflammation and amyloidosis. Colchicine is the gold standard treatment option, whereas anti-IL1 agents are reserved for patients with colchicine resistance or amyloidosis. ${ }^{17}$ Normal growth status brings us valuable information about well-being in children; therefore, growth monitoring is important in management of chronic diseases in childhood.

The first preliminary retrospective study investigating height in 17-year-old patients with FMF showed that colchicine-treated FMF patients had higher height than untreated patients although similar to normal population. ${ }^{18}$ Subsequently, a prospective study demonstrated similar height among 51 patients with FMF and healthy controls. Disease duration, attack frequency or ESR did not correlate with serum IGF-1 levels or height $\mathrm{SD}$ scores while there was a low-grade positive correlation between cumulative colchicine dosage and height velocity. Thus, it was speculated that colchicine improved growth by suppressing inflammation. ${ }^{5}$ At least, these studies suggested that colchicine does not deteriorate height velocity, even though it may improve growth in children with FMF.

Similarly, Zung et al. ${ }^{8}$ also revealed improvement in both height and weight SD scores after colchicine treatment. However, our study revealed lower height SD score in the posttreatment than the pretreatment period. This controversy cannot be solely clarified by our results because there may be other factors which may affect growth other than colchicine or disease itself, including parental height, nutrition or physical activity.

It was previously suggested that inflammatory cytokines, particularly IL-6, tumor necrosis factor-alpha, and IL-1 $\beta$, may affect growth hormone/insulin-like growth factor 1 axis and subsequently cause growth retardation. ${ }^{19}$ In another study; height and weight SD scores were found significantly lower in colchicineresistant FMF patients with elevated serum amyloid A levels than colchicine responders. Meanwhile, the growth retardation was attributed to sustained inflammation by the authors. ${ }^{6}$ Because colchicine-resistant patients were successfully treated with concomitant canakinumab in our study, we did not investigate colchicine resistance as a parameter on growth.

Furthermore, two independent retrospective studies with small numbers of FMF patients investigated the effects of genotype on growth parameters. Both demonstrated that homozygote M694V mutations had no impact on weight and height SD scores.7,8 On the contrary, we found significantly lower initial height SD score in patients with at least one M694V mutation than others. Furthermore, growth parameters at last visit did not differ between patients according to bearing a M694V mutation or not. We speculate that the negative impact of genotype on height can be alleviated with appropriate colchicine treatment. Researchers did not identify any effect of disease severity assumed by Tel Hashomer severity scoring system on growth in the latter study. ${ }^{8}$ Two recent studies also showed that disease severity was not correlated with growth parameters; however, these data were obtained from small numbers of patients. ${ }^{9,10}$

Disease severity assessment may be helpful in daily practice in terms of predicting the prognosis and guidance of patients. There are mainly two other severity scoring systems, conducted by Mor et al. ${ }^{20}$ and Pras et al., ${ }^{21}$ in addition to Tel Hashomer severity scoring system. The scoring system of Mor et al. ${ }^{20}$ consists of age at onset, colchicine dosage, 
number of sites involved during FMF attacks, pleuritic, and erysipelas-like erythema. On the other hand, the scoring system of Pras et al. ${ }^{21}$ includes the following six items: age at onset, colchicine dosage, attack frequency per month, presence of arthritis, erysipelas-like erythema, and amyloidosis. Tel Hashomer severity scoring system is almost the same as the system by Sohar et al. ${ }^{11}$ and Pras et al. ${ }^{21}$ except that it also contains phenotype II and divides arthritis into acute and protracted course. All aforementioned systems were designed for adults; however, they were seldomly used in pediatric patients without validation so far. Recently, one study compared the disease severity tools by Mor et al. ${ }^{20}$ and Pras et al. ${ }^{21}$ in pediatric FMF patients and found inconsistency between these two systems. Thus, a new disease severity tool, ISSF, has been proposed for pediatric FMF patients. ISSF consists of nine elements including chronic sequela (amyloidosis, growth retardation, anemia, and splenomegaly), organ dysfunction (FMF-related nephrotic range proteinuria), FMF-related organ failure, frequency of attacks, increased acutephase reactants, involvement of more than two sites during an individual acute attack, more than two different types of attack during the course of the disease, duration of attacks, and exertional leg pain. By introducing ISSF to the literature, this preliminary study detected significantly higher sensitivity and specificity than previous tools of Mor et al. ${ }^{20}$ and Pras et al. ${ }^{21}$ in both adults and children with FMF. However, the results were more reliable in adults than children. ${ }^{22}$ This can be attributed to the underestimation of clinical symptoms such as pleuritis, pericarditis, and myalgia; and also to the lack of chronic organ damages in pediatric patients. Since the items of Tel Hashomer severity scoring system were comparable with Pras et al. ${ }^{21}$ we still consider ISSF severity scoring system to be more eligible in children compared to the aforementioned scoring systems.

The limitations of our study were the retrospective design, lack of standardization of nutritional status, and small study sample. Whether other factors interfere with our results is up for discussion; however, our study is still worthsharing by being the first study investigating the relevance between growth and disease severity assessed by a validated disease severity scoring system in the largest FMF cohort so far. Further studies are needed to confirm our results.

In conclusion, we performed initial ISSF scoring to clarify whether disease severity affects growth in children with FMF. We found statistically lower initial height, final height, and weight SD scores in patients with intermediate severity-severe disease activity than those with mild disease. Moreover, we showed that although genotype has an impact on growth initially, disease severity may affect growth even at the end of follow-up. Thus, we suggest physicians to score disease severity prospectively and pay attention to patients with intermediate severity-severe FMF to avoid growth disturbances.

\section{Declaration of conflicting interests}

The authors declared no conflicts of interest with respect to the authorship and/or publication of this article.

\section{Funding}

The authors received no financial support for the research and/or authorship of this article.

\section{REFERENCES}

1. Ben-Chetrit E, Levy M. Familial Mediterranean fever. Lancet 1998;351:659-64.

2. Martorana D, Bonatti F, Mozzoni P, Vaglio A, Percesepe A. Monogenic Autoinflammatory Diseases with Mendelian Inheritance: Genes, Mutations, and Genotype/Phenotype Correlations. Front Immunol 2017;8:344.

3. de Jesus AA, Canna SW, Liu Y, Goldbach-Mansky R. Molecular mechanisms in genetically defined autoinflammatory diseases: disorders of amplified danger signaling. Annu Rev Immunol 2015;33:823-74.

4. Kasifoglu T, Bilge SY, Sari I, Solmaz D, Senel S, Emmungil $\mathrm{H}$, et al. Amyloidosis and its related factors in Turkish patients with familial Mediterranean fever: a multicentre study. Rheumatology (Oxford) 2014;53:741-5.

5. Savgan-Gürol E, Kasapçopur O, Hatemi S, Ercan O, Caliskan S, Sever L, et al. Growth and IGF-1 levels of children with familial Mediterranean fever on colchicine treatment. Clin Exp Rheumatol 2001;19(5 Suppl 24):S72-5.

6. Sozeri B, Yilmaz E, Mir S, Berdeli A. Effect of colchicine-resistant familial mediterranean fever on growth parameters. Arch Rheumatol 2011;26:1-6.

7. Türkmen $M$, Soylu $\mathrm{OB}$, Kasap B, Güneş $\mathrm{S}$, Tüfekçi $\mathrm{O}$, Soylu $\mathrm{A}$, et al. Growth in familial mediterranean 
fever: effect of attack rate, genotype and colchicine treatment. J PediatrEndocrinolMetab 2008;21:789-92.

8. Zung A, Barash G, Zadik Z, Barash J. Familial Mediterranean fever and growth: effect of disease severity and colchicine treatment. J Pediatr Endocrinol Metab 2006;19:155-60.

9. Yoldaş TÇ, Çakar N, Başaran Ö, Acar B, Uncu $\mathrm{N}$, Çaycı FŞ. The effect of colchicine and disease severity on physical growth in children with familial Mediterranean fever. Clin Rheumatol 2016;35:1603-7.

10. Ozçakar ZB, Kadioğlu G, Siklar Z, Kavaz A, Nur Aksanal F, Berberoğlu M, et al. The effect of colchicine on physical growth in children with familial mediterranean fever. Eur J Pediatr 2010;169:825-8.

11. Sohar E, Gafni J, Pras M. Tel Hashomer key to severity score for FMF. In: Sohar E, Gafni J, Pras $\mathrm{M}$, editors. Proceedings of the First International Conference of FMF. Tel Aviv: Freund Publishing House; 1997. p. 208.

12. Demirkaya E, Acikel C, Hashkes P, Gattorno M, Gul A, Ozdogan $\mathrm{H}$, et al. Development and initial validation of international severity scoring system for familial Mediterranean fever (ISSF). Ann Rheum Dis 2016;75:1051-6.

13. Neyzi O, Gunoz A, Furman A, Bundak R, Gökçay G, Darendeliler F, et al. Türk Çocuklarında vücut ağırlığı, boy uzunluğu, baş çevresi ve vücut kitle indeksi referans değerleri. Çocuk Sağlığı ve Hastalıkları Derg 2008;51:1-14.

14. Giancane G, Ter Haar NM, Wulffraat N, Vastert SJ, Barron K, Hentgen V, et al. Evidence-based recommendations for genetic diagnosis of familial Mediterranean fever. Ann Rheum Dis 2015;74:635-41.
15. Barut K, Sahin S, Adrovic A, Sinoplu AB, Yucel G, Pamuk G, et al. Familial Mediterranean fever in childhood: a single-center experience. Rheumatol Int 2018;38:67-74.

16. Tunca M, Akar S, Onen F, Ozdogan H, Kasapcopur O, Yalcinkaya F, et al. Familial Mediterranean fever (FMF) in Turkey: results of a nationwide multicenter study. Medicine (Baltimore) 2005;84:1-11.

17. Ozen S, Demirkaya E, Erer B, Livneh A, Ben-Chetrit $\mathrm{E}$, Giancane G, et al. EULAR recommendations for the management of familial Mediterranean fever. Ann Rheum Dis 2016;75:644-51.

18. Zemer D, Livneh A, Danon YL, Pras M, Sohar E. Long-term colchicine treatment in children with familial Mediterranean fever. Arthritis Rheum 1991;34:973-7.

19. MacRae VE, Wong SC, Farquharson C, Ahmed SF. Cytokine actions in growth disorders associated with pediatric chronic inflammatory diseases (review). Int $\mathrm{J}$ Mol Med 2006;18:1011-8.

20. Mor A, Shinar Y, Zaks N, Langevitz P, Chetrit A, Shtrasburg S, et al. Evaluation of disease severity in familial Mediterranean fever. Semin Arthritis Rheum 2005;35:57-64.

21. Pras E, Livneh A, Balow JE Jr, Pras E, Kastner DL, Pras M, et al. Clinical differences between North African and Iraqi Jews with familial Mediterranean fever. Am J Med Genet 1998;75:216-9.

22. Kalkan G, Demirkaya E, Acikel CH, Polat A, Peru H, Karaoglu A, et al. Evaluation of the current disease severity scores in paediatric FMF: is it necessary to develop a new one? Rheumatology (Oxford) 2012;51:743-8. 